Itinéraires Itinéraires

Littérature, textes, cultures

\title{
Les femmes et le comique dans le théâtre du marquis de Sade
}

Bénédicte Prot

\section{(2) OpenEdition}

Journals

Édition électronique

URL : http://journals.openedition.org/itineraires/746

DOI : 10.4000/itineraires.746

ISSN : 2427-920X

Éditeur

Pléiade

\section{Édition imprimée}

Date de publication : 1 mars 2014

Pagination : 153-165

ISBN : 978-2-343-02712-8

ISSN : $2100-1340$

\section{Référence électronique}

Bénédicte Prot, "Les femmes et le comique dans le théâtre du marquis de Sade », Itinéraires [En ligne], 2013-2 | 2014, mis en ligne le 01 novembre 2013, consulté le 19 avril 2019. URL : http://

journals.openedition.org/itineraires/746 ; DOI : 10.4000/itineraires.746

\section{(ब) $(\Theta \Theta$}

Itinéraires est mis à disposition selon les termes de la licence Creative Commons Attribution - Pas d'Utilisation Commerciale - Pas de Modification 4.0 International. 


\section{Les femmes et le comique dans le théâtre du marquis de Sade}

\section{Résumé}

Cet article étudie la place et la fonction des femmes dans les fictions de Sade, et plus précisément dans son théâtre. L'enjeu consiste à analyser le rôle des personnages féminins dans trois de ses comédies (Le Boudoir, Les Jumelles, Le Misanthrope par amour). Ces figures révèlent ce qui intéresse Sade dans l'écriture dramatique : alors que ce volet de son œuvre est traditionnellement déconsidéré, il s'agit de valoriser son comique spécifique et ses liens avec les héroïnes de ses pièces.

Mots clés : théâtre de Sade, comédie, philosophie, femmes, ironie

\section{Abstract}

This article investigates the place and function of women in Sade's fictions and focuses specifically on Sade's theatre. Its aim is to define the role of female characters in three comedies (Le Boudoir, Les Jumelles, Le Misanthrope par amour). The characters in question show Sade's intentions in his theatre: we contend that Sade is playing with the rules of traditional dramatic art, and that he aims, as a result, at creating a particular comical effect. Sophie, Mme Dolcour, Adélaïde and Julie are not stereotypes but philosophical entities. They reveal how Sade exposes his preoccupations on stage and how he attempts to create a comic based on irony and imagination.

Keywords: Sade's theatre, comedy, philosophy, women, irony

En 1979, Michel Delon faisait le bilan de « Dix ans d'études sadiennes » et concluait : «C'est finalement comme discours double qu'il faut comprendre le discours sadien sur les femmes : discours misogyne dominant, discours féministe cohérent et minoritaire ${ }^{1} »$. Cette alternative faisait des héroönes sadiennes tantôt les victimes d'un système intolérable, tantôt des scélérates aguerries. Les femmes du théâtre sadien sont en dehors de cette typologie, 
certainement parce qu'on peine à reconnaître la plume du marquis dans des pièces dont on ne retient bien souvent que la médiocrité. Considérant que « [b]eaucoup a été fait pour arracher Sade à la censure et au silence, beaucoup demeure à faire pour le libérer du mythe ${ }^{2}$ ", nous proposons d'étudier les figures féminines de ce théâtre afin d'envisager ailleurs et autrement la question de la féminité chez Sade et de redonner au corpus dramatique sa place et sa pertinence au sein de l'œuvre du marquis.

Les comédies semblent bien éloignées des préoccupations du Sade romancier, comme en témoignent des titres comme Le Capricieux, Le Prévaricateur, Le Philosophe soi-disant ou encore Les Antiquaires. Trois pièces que Sade qualifie de comédies retiennent notre attention : Le Misanthrope par amour (1781-1782), Le Boudoir (1783) et Les Jumelles (17801781). Le Misanthrope par amour présente l'histoire de l'amour contrarié entre Sophie, qui croit aimer son tuteur, et Desfrancs, qui devient misanthrope à force d'aimer celle qu'il croit être sa fille naturelle. Le Boudoir met en scène un cours de morale entre Mme Dolcour et son amant, afin de lever les doutes d'un M. Dolcour jaloux au point d'épier son épouse pour s'assurer de sa fidélité. Enfin, l'intrigue des Jumelles repose sur l'arrivée du jeune Damis qui doit choisir celle qu'il épousera entre deux sœurs parfaitement identiques.

Sans qu'il soit question de réhabiliter ces fameux « rejetons disgraciés $^{3} \gg$ que le marquis aurait écrits par passion pour le théâtre, le constat s'impose de l'importance et de l'omniprésence des figures féminines. Sade fait en outre preuve d'une maîtrise des principes et des règles de la dramaturgie. Des titres comme Le Boudoir, Les Jumelles et Le Misanthrope par amour, souvent titré Sophie et Desfrancs, mettent l'accent sur les figures féminines. Les intrigues nous invitent du côté de «l'imitation des mours, mise en action ${ }^{4}$ » et suggèrent la mise en scène d'un caractère théâtral, comme dans Le Misanthrope par amour, et même Le Boudoir, parfois intitulé L'École des jaloux, Le Mari crédule, ou La Folle Épreuve. Dans Les Jumelles, Marton donne le ton d'une comédie qui raille les maîtres: «Les défauts sont leur lot et le nôtre est d'en rire ${ }^{5}$ ». Les femmes s'inscrivent dans des intrigues redevables d'une tradition comique dans laquelle on rit des mœurs et des caractères du temps et par laquelle on cherche à perfectionner l'éducation morale du spectateur.

La question du genre se décline ici sous deux aspects : il s'agit d'interroger les figures féminines selon leur mise en scène dans un répertoire comique dont on observera les règles de composition - le déroulement de l'intrigue

2. Michel Delon, « Dix ans d'études sadiennes », op. cit., p. 426.

3. Gilbert Lely, Vie du marquis de Sade, Paris, Pauvert, 1965, p. 409.

4. Jean-François Marmontel, Éléments de littérature, s. 1., 1787, vol. II, p. 138.

5. Sade, Les Jumelles, dans CEuvres complètes, Paris, Pauvert, 1970, t. XXXII, acte I, sc. II, p. 298. 
dans l'univers du quotidien, le dénouement moral et bienséant - ainsi que les règles de traitement des personnages - la condamnation du caractère et des travers, le ridicule. Comment Sade construit-il ses personnages féminins, ayant pleine connaissance des normes du genre comique, tant représentatives que morales? Dans quelle mesure ces femmes contribuent-elles aux enjeux du théâtre et du comique sadiens? Répondre à ces questions dévoilera la richesse des personnages féminins comiques de Sade. Croyons donc le marquis sur parole quand il concède qu' « on a déjà bien parlé des femmes... [...] et il serait possible que l'on n'eût pas encore dit sur leurs qualités tout ce qu'il y avait à dire, ce fond est comme leurs grâces... inépuisable ${ }^{6}$ ».

\section{Un jeu sur les procédés dramatiques de la comédie}

D'aucuns se sont intéressés au " grand mal-aimé de notre littérature ${ }^{7}$ », affirmant que l'intérêt de ce théâtre, tout conventionnel qu'il est, réside dans le jeu que Sade opère sur les procédés dramatiques. Les études de Sylvie Dangeville et de Thomas Wynn ${ }^{8}$ ont ainsi permis de rattacher le théâtre au reste de l'œuvre, tant par l'analyse des pièces que par celle de l'esthétique du spectacle sadien. Pierre Frantz traite de la question du genre dans ce théâtre et attire notre attention sur « la position du joueur qui commande la manière dont le marquis se saisit des éléments génériques du théâtre ${ }^{9} »$. La construction du personnage est ici un point fondamental du genre comique avec lequel Sade se plait à jouer.

Les premières scènes des pièces donnent peu d'informations sur ces personnages féminins et leurs portraits in absentia laissent apercevoir de bien pâles figures de comédie. Dans Le Misanthrope par amour, nous savons seulement de Sophie, avant son entrée en scène, qu'elle est « [j]eune, belle et sensible et que chacun [1']envie ${ }^{10} \gg$. Ses premières apparitions témoignent de sa vivacité, trait sur lequel Sade insiste par ses didascalies à la scène III de l'acte I. Sophie nous apparaît au premier abord comme une jeune femme naïve et spontanée, fidèle au portrait que Desfrancs esquisse : « Elle est sans art, c'est la simple nature ${ }^{11} \gg$. La femme du Boudoir ne semble pas avoir de traitement plus fouillé. M. Dolcour interroge sa servante, soupçonnant quelque infidélité entre son épouse et son cousin Sérigny. Avant même son

6. Sade, Avant-propos des Jumelles, dans CEuvres complètes, op. cit., p. 289-290.

7. Maurice Lever, préface, dans Sylvie Dangeville, Le théâtre change et représente : lecture critique des euvres dramatiques du marquis de Sade, Paris, Champion, 1999, p. 11. 8. Thomas Wynn, Sade's Theatre: Pleasure, Vision, Masochism, Oxford, Voltaire Foundation, 2007.

9. Pierre Frantz, «Les caractères dans le théâtre de Sade : la loi du genre », Annuaire théâtral : Revue québécoise d'études théâtrales, $\mathrm{n}^{\circ}$ 41, 2007, p. 20.

10. Sade, Le Misanthrope par amour, dans Euvres complètes, Paris, Pauvert, 1970, t. XXXV, acte I, sc. II, p. 35.

11. Ibid., acte II, sc. II, p. 66. 
entrée en scène, Mme Dolcour est suspectée de «trop de coquetterie ${ }^{12}$ ». Le portrait que Lucile fait de sa maîtresse, trop élogieux pour être vrai, est éloquent :

\author{
Florval la lutinait, elle était insensible, \\ Alceste cajolait, elle était inflexible, \\ À Célimène enfin, qu'elle adore aujourd'hui, \\ Il ne fut même pas possible \\ De pouvoir d'elle obtenir un seul oui. \\ Insouciante, inaccessible ${ }^{13}$.
}

Ce contre-portrait hyperbolique servant à flouer le pauvre jaloux est démenti par l'attitude pleine de coquetterie de Mme Dolcour dès son arrivée : «Elle est dans le déshabillé le plus coquet et le plus élégant, de la négligence dans la démarche et un peu de langueur dans le débit ${ }^{14} »$. Elle poursuivra en s'exprimant avec « le froid le mieux gazé, et bien de l'art », " puis avec négligence » enfin avec « [p]lus de minauderie que de vérité ${ }^{15} \gg$. Pour ce qui est des jumelles Julie et Adélaïde, leurs caractéristiques restent somme toute très sommaires. Sade insiste sur leur grande ressemblance dans un portrait physique qui regroupe les deux jeunes femmes. Nous savons peu de chose sur leurs complexions : elles se distinguent par le simple fait que " [1]'une pourtant, Julie, a moins de gravité / Et plus qu'Adélaïde a d'ingénuité ${ }^{16}{ }^{\prime}$. Une jeune naïve, des jumelles sans particularités et une simple coquette: tels semblent les personnages féminins ébauchés dans ces pièces. Aucune complexité de caractère ni de véritable psychologie ne semble intéresser le marquis dans la création de ces femmes de comédie. De surcroît, on ne saurait ignorer les emprunts que Sade fait à la tradition comique et à l'univers dramatique du siècle : la coquette est depuis Molière un personnage type de la comédie, tout comme Sade ne cache pas que Plaute, Molière et Regnard l'ont inspiré pour ses Jumelles. Enfin, cette jeune naïve qu'est Sophie peut aisément nous faire penser à l'héroïne du Père de famille, drame bourgeois que Sade admirait au point de confier le rôle phare à Léonore, qui débute sa carrière de comédienne à la fin d'Aline et Valcour.

Difficile pourtant de s'en tenir à ce manque d'audace et à cette «psychologie superficielle ${ }^{17}$ " qu'Anne Lacombe attribue à la plupart des personnages de la dramaturgie sadienne. Pierre Frantz affirme que Sade « refuse l'héritage d'un type, défini dans la comédie classique par

12. Sade, Le Boudoir, dans Euvres complètes, Paris, Pauvert, 1970, t. XXXIII, sc. I, p. 103.

13. Ibid., p. 102.

14. Ibid., sc. II, p. 114.

15. Ibid., p. 115-116.

16. Sade, Les Jumelles, op. cit., acte I, sc. II, p. 302.

17. Anne Lacombe, « Du théâtre au roman: Sade », Studies on Voltaire and the eighteenth century, $\mathrm{n}^{\circ} 129,1975$, p. 128. 
l'hypertrophie d'une passion dans un caractère ${ }^{18} »$ et nous invite à aller au-delà de l'apparente fadeur des personnages. Suivons Sade lorsqu'il affirme que : «Ce n'est pas toujours la figure principale qu'il faut toucher pour la faire ressortir, le plus souvent ce sont celles qui l'entourent ${ }^{19} \gg$. L'illusion d'une comédie traditionnelle avec ses types et ses questionnements habituels - le mariage, l'amour - demeure, mais ce sont bel et bien les personnages féminins qui, à défaut d'être de véritables caractères de théâtre, jouissent d'une envergure philosophique inédite.

Dans Le Misanthrope par amour, Sade nous prévient de la complexité du rôle de Desfrancs alors que « [1]e personnage de Sophie, quelque violent qu'il soit, n'appartient décidément qu'aux ingénuités ${ }^{20} »$. L' ' Avertissement » de la pièce nous précise que « ce n'est point ici une coquette, une de ces filles artificielles, soigneusement élevées dans des cloîtres et dans le caractère desquelles on s'étudie à remplacer par de la fausseté tous les sentiments que le cœur inspire ${ }^{21} \gg$. C'est moins un trait psychologique qu'une conformation philosophique qui se révèle derrière l'étonnante simplicité de Sophie. Le dramaturge conçoit son personnage selon des principes qui traversent toute son œuvre :

Sophie est une fille ingénue, une espèce de sauvage : elle est l'enfant de la nature, elle ne connaît que cette voix et celle de son cœur; elle s'habille mais elle ne se pare point, elle veut plaire mais elle n'a dessein ni de tromper ni de séduire; le climat sous lequel elle est née donne un degré d'activité terrible à des passions qu'elle croit naturelles... parce qu'elles le sont et qu'excepté la candeur, le devoir filial, la bienfaisance et l'humanité elle ne connaît pas d'autres vertus ${ }^{22}$.

C'est cette disposition qui distingue Sophie du misanthrope « par circonstance ${ }^{23} \gg$ et lui fait incarner la voix de la nature. Au cours des actes III et IV, elle va affirmer son attachement pour son tuteur - «Non, rien n'arrachera Desfrancs de sa Sophie / Pour toujours avec lui je veux passer ma vie ${ }^{24} \gg-$ et niera toute culpabilité pour des sentiments qu'elle considère naturels : « Je devais avouer cet amour légitime, / Il n'est aucun mal à l'aimer ${ }^{25}$ ». La jeune femme fait preuve d'une grande fermeté en refusant les discours autoritaires de Desfrancs - « N'espérez pas me vaincre ${ }^{26}$ » - et fait renoncer Anselme à des projets de mariage dont elle « jure / De ne jamais suivre

18. Pierre Frantz, « Sade : texte, théâtralité », dans Michel Camus et Philippe Roger (dir.), Sade, écrire la crise, Paris, Belfond, 1983, p. 196.

19. Réflexions sur Le Prévaricateur, dans Euvres complètes, op. cit., t. XXXV, p. 278.

20. Avertissement au Misanthrope par amour, op. cit., p. 22.

21. Ibid., p. 23.

22. Ibid., p. 23-24.

23. Ibid., p. 21.

24. Ibid., acte III, sc. III, p. 86.

25. Ibid., acte IV, sc. I, p. 102.

26. Ibid., acte III, sc. III, p. 84. 
la $1 \mathrm{loi}^{27} \gg$. Sophie pose la question du mariage forcé de la même manière que Sade se demande, dans La Philosophie dans le boudoir, s'il est « au monde, $[\ldots]$ un sort plus affreux que celui-là ${ }^{28} »$. Alors que Desfrancs annonce une séparation inévitable, la réaction de sa fille sera de « rejet[er] cette idée comme horrible pour elle ${ }^{29} »$ jusqu'à être « dans le plus violent état $^{30} »$. C'est au cœur même d'une comédie que Sophie incarne la nature et le rapport conflictuel entre les lois naturelles - un amour incestueux - et les lois sociales. Étrange comédie qui prétend faire rire des caractères et dans laquelle la figure féminine possède un fond philosophique, miroir de la pensée de Sade.

Dans Le Boudoir, Mme Dolcour est mise en scène face à son mari, dont le travers doit être corrigé. Si Mme Dolcour n'est pas la première coquette de la scène comique, il semble bien qu'elle puisse produire « des impressions faites pour alarmer les amis des bonnes mœurs ${ }^{31} »$. Cela va sans dire : à la différence de son mari, elle possède un fond philosophique incontestable et persifle toutes les valeurs établies. Son attitude de rouée n'est pas sans rappeler Mme de Saint-Ange : toutes deux mises en scène dans un boudoir, elles s'illustrent par un cours de morale libertine qui débute par le refus de l'amour: « Mais je n'adopte ces chimériques flammes / Et ce qui les allume est un besoin des sens / Que très aisément on maîtrise ${ }^{32}$ ». Pendant cette leçon qu'elle simule avec Sérigny, Mme Dolcour se livre à une condamnation toute hypocrite et hyperbolique de l'amour qui reste « $[u] n$ sentiment qui nous déprave » et qui «a brisé plus de freins / Que tous les crimes de la terre ${ }^{33} \gg$. En outre, elle fournit en quelque sorte la réponse concrète à la question de la jeune Eugénie dans La Philosophie dans le boudoir: " Presse-toi donc en ce cas de m'apprendre quelle doit être la conduite d'une femme dans le mariage ${ }^{34}$ ? » Mme Dolcour incarne sur scène l'art de vivre libertin : par sa fausseté tout au long des scènes, elle démontre « comme un peu d'art, un peu d'inconséquence / soumet [les hommes] en leur résistant ${ }^{35} \gg$. Face à cette femme qui joue la comédie de la pruderie et du moralisme austère, le mari devient première victime du fameux adage de Mme de Saint-Ange : « Je foutrais avec toute la terre,

27. Ibid., acte III, sc. I, p. 79.

28. Sade, La Philosophie dans le boudoir, dans Euvres, éd. Michel Delon, Paris, Gallimard, coll. «Bibliothèque de la Pléiade », t. III, 1998, p. 39.

29. Sade, Le Misanthrope par amour, op. cit., acte III, sc. III, p. 85.

30. Ibid., p. 87.

31. Lettre de Miramond à Sade à propos du Boudoir, dans Euvres complètes, op. cit., p. 90 .

32. Sade, Le Boudoir, op. cit., sc. XI, p. 149.

33. Ibid., p. 151.

34. Sade, La Philosophie dans le boudoir, op. cit., p. 39.

35. Sade, Le Boudoir, op. cit., sc. III, p. 122. 
sans lui faire une égratignure ${ }^{36}$ ». Dans cette prétendue « jolie bluette ${ }^{37}$ » qui oppose un jaloux et sa femme, Mme Dolcour ne peut que surprendre par ses aspirations philosophiques.

Dans Les Jumelles, si l'on ne sait presque rien des deux sœurs, Damis est l'exemple même du jeune galant et Durval, amoureux d'Adélaïde, est un amant fidèle et honnête. Tout porte à croire que les deux sœurs doivent, selon le schéma traditionnel, corriger le défaut du jeune volage. Mais l'extrême ressemblance des jumelles va plus loin et ne saurait se réduire à un simple rouage comique riche de quiproquos : considérées comme des « fleurs offertes [aux] sensations ${ }^{38}$ » des jeunes gens, les jumelles incarnent la pensée sensualiste et érotique de Sade, dans laquelle les sens sont le moteur du désir toujours fluctuant. Damis est en effet soumis à deux beautés dont les " mêmes traits, [le] même esprit / Jusqu'au son de la voix, tout en elles séduit / Trompe, embarrasse ${ }^{39} \gg$. La comédie galante des jeunes femmes est orchestrée par leur mère, qui refuse absolument de leur imposer un mari. Ces deux comédiennes soulèvent alors la question de la condition féminine, qui est d'ailleurs disputée entre Damis et Durval. Chacun aborde une conception de la femme différente et l'on peine à savoir qui des deux trouve raison à la fin de la pièce. Il semble pourtant que les jumelles y répondent en se considérant comme "parfaitement interchangeables ${ }^{40}$ », selon les termes de Sylvie Dangeville. Julie et Adélaïde gardent possible leur échange et restent ainsi un éternel objet de désir, jamais soumis au choix des hommes ni « [a]ux rigoureux liens de [leur] dépendance ${ }^{41} »$. Les jumelles disposent de leur sort tant le «choix difficile» de Damis est en réalité impossible : «Ces deux sœurs-là jamais ne formeront deux filles / Également pensant, également gentilles / À tel point qu' on ne peut faire entre elles de choix ${ }^{42}$ ». Sylvie Dangeville classe ainsi Les Jumelles dans « le théâtre de l'inceste », et Marie-France Silver y voit une preuve du « féminisme » de Sade, remarquant que le marquis « dote ses héroïnes de capacités rarement associées avec le sexe faible ${ }^{43} \gg$ dans la représentation de ces deux sœurs qui s'affranchissent des lois du mariage par leur jeu de ressemblance. On comprend dès lors à quel point les femmes des Jumelles produisent « un effet sûr et peut-être même entièrement neuf ${ }^{44} \gg$ sur la scène comique.

36. Sade, La Philosophie dans le boudoir, op. cit., p. 41.

37. Sade, Opuscules sur le théâtre, dans CEuvres complètes du marquis de Sade, Paris, Tête de Feuilles, 1973, t. XVI, p. 471.

38. Avant-propos des Jumelles, op. cit., p. 290.

39. Sade, Les Jumelles, op. cit., acte I, sc. II, p. 302.

40. Sylvie Dangeville, Le théâtre change et représente, op. cit., p. 183.

41. Sade, Les Jumelles, op. cit., acte I, sc. X, p. 322.

42. Ibid., acte II, sc. VI, p. 339.

43. Marie-France Silver, "Le théâtre du marquis de Sade », dans Le Théâtre dans l'Europe des Lumières. Programmes. Pratiques. Échanges, Actes du colloque organisé par le Centre d'Études Littéraires Comparées de l'Université de Wroclaw, Karpacz, 18-22 avril 1983, p. 177. 44. Avant-propos des Jumelles, op. cit., p. 287. 
Ces personnages de femmes relèvent davantage d'une constitution philosophique que d'un type comique. Elles contreviennent aux lois sociales, tout comme d'autres héroïnes du théâtre sadien ont elles aussi retenu l'attention : c'est le cas de Jeanne Laisné, que Michel Delon rapproche de Juliette et d'Isabelle de Bavière par son énergie extraordinaire ${ }^{45}$, ou encore d'Ernestine, dans Oxtiern, dont on peut remarquer la vigueur et la force de caractère ${ }^{46}$. Les femmes de ce théâtre comique portent la réflexion philosophique de Sade et sa réflexion ambiguë sur la condition féminine : la femme dépouillée des préjugés est bien souvent d'une vertu douteuse. Ces figures comiques participent ainsi au détournement des problématiques apparentes des pièces - corriger un jaloux, un misanthrope et un volage - et malmènent la vocation morale du genre. Rien ne nous surprend dans le fait que ces pièces ne furent jamais représentées : le marquis offre à ces femmes la possibilité de renouveler les sujets du registre comique selon sa propre conception des mœurs et sa pensée si singulière.

\section{Comique sadien et représentation de la féminité}

Nous pouvons d'ores et déjà nuancer l'hypothèse d'un moindre intérêt du théâtre de Sade. La présence de ces figures féminines explique en grande part qu'il n'y ait, comme l'analyse André Guyaux, « aucun effet de comique $[\ldots]$ réussi ${ }^{47} \gg$. Leur mise en scène dans des pièces où la notion de caractère est flottante, et où les personnages évoluent dans « une suite naturelle d'évé[ne]men[t]s familiers ${ }^{48} »$, tout en abordant de manière surprenante des questionnements convenus, rend la plaisanterie du dramaturge plus sérieuse. Sade obtient, grâce à ces héroïnes, un effet comique singulier : à l'évidence, la représentation de la féminité est liée à la création du « haut comique ${ }^{49}$ » et de « la fine plaisanterie ${ }^{50} »$, auxquels le marquis dramaturge prétendait. La comédie au XVIII ${ }^{\mathrm{e}}$ siècle s'assigne toujours un objectif ambitieux : montrer le vice pour le corriger et atteindre la morale et la vertu. Sade a bien compris cette démarche et s'y livre à cœur joie dans toute son œuvre : « L'art [est] établi pour être l'école des mœurs ${ }^{51}$ », à condition de « permettre au vice de parler sa langue, si l'on veut voir le vice puni au théâtre ${ }^{52} \gg$. Or, les femmes du théâtre sadien inaugurent un comique où le vice s'exprime avec hardiesse sur scène.

45. Michel Delon, « Jeanne Laisné, hérö̈ne sadienne », dans Paul Mironneau et Gérard Lahouati (dir.), Figures de l'histoire de France dans le théâtre au tournant des Lumières (1760-1830), Oxford, Voltaire Foundation, coll. «SVEC», nº 7, 2007, p. 81-88.

46. Voir Jean-Jacques Roubine, « Oxtiern, mélodrame et palimpseste», Revue de la société d'histoire du théâtre, $n^{\circ} 22,1970$, p. 266-283, et Marie-France Silver, op. cit.

47. André Guyaux, "Théâtre de Sade », Revue d'histoire du théâtre, n 121, 1979, p. 47.

48. Jean-François Marmontel, Éléments de littérature, op. cit., p. 145.

49. Avertissement au Misanthrope par amour, op. cit., p. 23.

50. Sade, Opuscules sur le théâtre, op. cit., p. 471.

51. Réflexions sur Le Prévaricateur, op. cit., p. 273.

52. Ibid., p. 280. 
Mue par une passion qu'elle croit naturelle, Sophie va manifester les délires et les mécanismes violents d'un amour d'abord contrarié par le refus de Desfrancs, puis rejeté comme horrible en raison de son caractère incestueux. Pendant les actes IV et V, son comportement nous est décrit par les didascalies qui évoquent de nombreux épanchements, des pleurs, des pertes des sens : ce délire relève d'un pathos incontestable et doit produire « un effet déchirant, et vraiment théâtral ${ }^{53} »$. On imagine cette figure féminine éplorée, d'une violence et d'un pathétique exacerbés, supposée montrer le vice dans toute son horreur et le désespoir de la nature contrariée. C'est donc avec une certaine emphase que Sade aborde ici le thème de l'inceste, difficilement exploitable sur la scène comique. C'est pourtant bien ce à quoi il aspire : faire une comédie où le vice, sa violence et ses rouages, s'exposent de manière éclatante, voire grandiloquente.

Dans Les Jumelles, le vice est mis à nu par un processus scénique différent. Il ne s'agit pas ici d'opérer une distinction entre les jeunes filles, dont l'une pourrait être vertueuse et l'autre pas. L'antagonisme entre les deux sœurs n'intéresse pas Sade, à la différence de ce qui se passe dans son diptyque romanesque avec Justine et Juliette. La vertu n'est à proprement parler pas incarnée, puisque les jumelles se plient également à un jeu de séduction aussi plaisant que condamnable. L'acte II réunissant Adélaïde et Julie nous informe que « [1] es deux sœurs sont toutes deux très élégamment, très uniformément mises, on ne doit pas apercevoir la plus légère différence dans leur habillement ou leur coiffures ${ }^{54} »$. Cet « [é]trange effet d'optique lorsqu'un miroir ne renvoie qu'à un autre miroir ${ }^{55} \gg$ a pour but de séduire aussi bien Damis que le spectateur. Particulièrement dans Les Jumelles, Sade fait l'économie de la représentation de la vertu et insiste sur les traits séduisants du vice car «l'homme n'est pas incorrigible et si la vertu ne le séduit pas, c'est qu'elle lui est rarement présentée sous les véritables traits qui lui conviennent ${ }^{56} \gg$. Enfin, dans Le Boudoir, la représentation du vice se fait explicite et passe par le recours à l'érotisme. Mme Dolcour va faire de la scène le théâtre des plaisirs qu'elle partage avec son amant :

Eh! Monsieur, c'est selon

Comme l'enthousiasme a d'effet sur leur âme;

En s'y mettant d'abord, l'entretien est fort bas;

Par degrés il s'élève, et souvent il s'enflamme

En terminant tous leurs débats,

Au point qu'ils font un bruit qui ne se conçoit pas ${ }^{57}$.

53. Sade, Opuscules sur le théâtre, op. cit., p. 471.

54. Sade, Les Jumelles, op. cit., acte II, sc. I, p. 325.

55. Pierre Frantz, « Sade : texte, théâtralité », op. cit., p. 199.

56. Avant-propos des Jumelles, op. cit., p. 290.

57. Sade, Le Boudoir, op. cit., sc. I, p. 112. 
Le cours de morale de Mme Dolcour devient à n'en pas douter un jeu érotique pendant lequel «tous deux s'enthousiasment à l'envi l'un de l'autre », Sérigny se montrant « livré et sans plus de contrainte » et au terme duquel « [i]ls sont dans les bras l'un de l'autre ${ }^{58}$ ». C'est dans toute sa dimension physique que Sade nous présente ici le vice dans les jeux de scènes qui composent le rôle de la libertine Mme Dolcour.

Fille pathétique, sœurs séductrices et femme érotique : il s'agit bien, par ces figures, de produire une représentation du vice sur scène dans ce qu'il a de plus évident, " soit en propos soit en action ${ }^{59}$ ». Néanmoins, nous n'ignorons pas l'ambiguïté de la démarche sadienne qui prétend, par la peinture du vice, à « l'une des plus sublimes leçons de morale que l'homme ait encore reçue ${ }^{60} »$. Aussi, dans le registre comique, ce sont les destinées de ces femmes explicitement vicieuses qui créent cette ironie particulière dont le marquis a le secret.

Force est de constater tout d'abord que Sade s'abstient de ridiculiser ses femmes de comédie. Selon les exigences morales du genre, le dramaturge devrait condamner par le rire les penchants incestueux de Sophie, le libertinage de Mme Dolcour et la frivolité des jumelles. Or la femme adultère du Boudoir n'est blâmée ni pour son attitude, ni pour ses principes libertins, et les contemporains de Sade ne s'y sont pas trompés en faisant remarquer " qu'un pareil sujet exigerait encore plus de gaieté ${ }^{61} \gg$. Mme Dolcour garde le beau rôle et le comble du ridicule est atteint par le mari, qui la prie de s'unir avec Sérigny : « S’il est vrai qu'un époux ait des droits sur sa femme. / Au nom de nos liens, je veux que Sérigny / Pour jamais avec vous soit libre et réuni ${ }^{62} \gg$. On rit moins de la jalousie que du triomphe ironique et immoral de l'infidèle, qui trompe son mari sous ses yeux. Dans Le Misanthrope par amour, Sade fait dire à Desfrancs, non sans moquerie : « J'aimais en père et je dois maintenant / Apprendre à vos genoux à n'aimer qu'en amant ${ }^{63}$ ». À aucun moment Sophie n'est raillée pour sa passion incestueuse. Par sa proximité avec l'héroïne du Père de famille dans ses élans sensibles et touchants, elle doit nous paraître plus attendrissante que ridicule et ainsi permettre de donner à la pièce cette tournure dérangeante. Bien entendu, dans Les Jumelles, ni Julie ni Adélaïde ne sont l'objet d'un rire moqueur : comme nous l'annonce Marton au début de la pièce, «le choix, vraiment le choix, c'est le choix qui fait rire ${ }^{64} \gg$. Damis, ne sachant plus où donner de la tête, fait les frais du comique, tandis que les jumelles sortent indemnes de leur jeu de galanterie.

58. Ibid., sc. XI, p. 152.

59. Réflexions sur Le Prévaricateur, op. cit., p. 280.

60. Sade, Justine ou les Malheurs de la vertu, dans Euvres, éd. Michel Delon, Paris, Gallimard, coll. « Bibliothèque de la Pléiade », t. II, 1995, p. 130.

61. Lettre de Miramond à Sade à propos du Boudoir, op.cit., p. 90.

62. Sade, Le Boudoir, op. cit., sc. XII, p. 156.

63. Sade, Le Misanthrope par amour, op. cit., acte V, sc. VI, p. 139-140.

64. Sade, Les Jumelles, op. cit., acte I, sc. II, p. 301. 
Ce refus du ridicule fait, au dénouement des pièces, la part belle à ces femmes à la vertu chancelante. Alors que l'on s'attend au rétablissement de l'ordre vertueux, le triomphe des héroïnes referme les pièces sur une sorte d'ironie du sort. Mais chez le marquis, bafouer la vertu et la morale relève d'une interrogation plus complexe. Si ces femmes malmènent les bienséances du genre, ce sont aussi des êtres de spectacle qui créent sur scène des systèmes illusoires et utopiques auxquels Sade a toujours fait appel. Ainsi, dans Le Misanthrope par amour, lors d'une scène de reconnaissance artificielle et prévisible, Sophie recouvre l'usage de ses sens et peut désormais épouser Desfrancs. Ce dénouement convenu et soudain ouvre la pièce sur un nouvel horizon. La jeune femme ne retrouve la raison qu'au moment où Le Misanthrope par amour réalise, sous nos yeux, un monde où les lois humaines ne viennent plus contrarier celles de la nature. Sophie s'épanouit, chez Sade, dans toute sa dimension de personnage utopique, ressort d'une société créée de toutes pièces sur la scène de la comédie et dans laquelle « [r]ien n'est moins fort que la raison / Rien ne l'est plus que la nature ${ }^{65} \gg$.

Même cas de figure chez Mme Dolcour. Toute la pièce fonctionne sur la thématique de l'illusion théâtrale, dont la seule victime est M. Dolcour : alors que celui-ci voudrait révéler la réalité de la conduite de son épouse, celle-ci fait en sorte qu' « [e]n vérité rien ne se voi[e] ${ }^{66} »$. Les faux-semblants de Mme Dolcour relèvent moins d'une critique des femmes que de la création sur scène d'un monde où le libertinage s'exprime sans entrave. L'infidèle a d'ailleurs tout intérêt à souhaiter que « [s]ans que l'honneur s'effarouche et s'offense, / Sans s'écarter des bornes du devoir, / Puissent finir ainsi sans imprudence / Toutes les scènes de boudoir ${ }^{67} \gg$. On ne s'étonne guère ici que le lieu-même de la féminité devienne la scène utopique des démonstrations de libertinage les plus outrées et les moins réprimées. Aucune surprise non plus à ce que Le Boudoir, « dont le fond n'est point conforme aux règles de la bienséance ${ }^{68} »$, ait essuyé de nombreux refus ${ }^{69}$ : il trouvera sa forme la plus aboutie dans le roman dialogué du marquis.

Enfin, dans Les Jumelles, Julie et Adélaïde se contentent de la situation équivoque que leur ressemblance instaure au sein de la famille. Damis, pourtant charmé par Adélaïde, épousera par défaut sa sœur. Rien n'étonne les jumelles dans les dernières paroles du galant quand il dit à Julie : «Quoi qu'en vous épousant / (Montrant Adélaïde). Mon cœur lui rend hommage / Et je vais l'adorer sous sa plus belle image ${ }^{70} \gg$. Les deux sœurs présentent

65. Sade, Le Misanthrope par amour, op. cit., acte V, sc. v, p. 129.

66. Sade, Le Boudoir, op. cit., sc. VIII, p. 142.

67. Ibid., couplets, p. 159.

68. Lettre de Miramond à Sade à propos du Boudoir, op. cit., p. 90.

69. Pour preuve les nombreuses lettres que Sade écrit jusqu'en 1793 pour que sa pièce soit représentée, en vain.

70. Sade, Les Jumelles, op. cit., acte II, sc. x, p. 351-352. 
l'incroyable particularité d'être réduites à ne former «qu'un être à double face ${ }^{71} »$, manière pour Sade de nier l'individualité de chacune au sein de la famille. En effet, les remarques graveleuses des domestiques à la fin de la pièce scellent le sort des jumelles : « Ce soir au moins, Monsieur, n'allez pas vous tromper ${ }^{72}$ » et : «Combien d'époux voudraient pour calmer leur ennui / Que leur femmes de jour se changeassent les nuits ${ }^{73}$ ? \La possibilité d'éternelles confusions entre les deux jeunes filles perdure aussi bien pour les spectateurs que pour les autres personnages. La comédie se clôt sur une « illusion complète ${ }^{74}$ », dans laquelle Julie et Adélaïde incarnent le fantasme de Sade d'une société où l'individualité s'annule au profit de la loi du désir.

Les figures féminines de ces comédies créent sur scène un système illusoire et immoral : leur caractère utopique relève du comique sadien, qui repose sur la création d'un imaginaire pour mieux se gausser des principes établis et des préjugés. Le genre, sous la plume de Sade, perd sans doute ses ressorts efficaces - par le refus du ridicule - et sa portée morale - par l'expression outrée du vice et l'utopie qui conforte le libertinage et l'inceste. Mais il n'en perd pas pour autant sa portée critique : les jumelles, Mme Dolcour et Sophie nous rappellent que la mise en spectacle de la femme est bien souvent garante, chez Sade, de l'ironie qui caractérise son œuvre. Ces figures, qui relayent la comédie dans un univers utopique, sont les parfaits rouages de toute fiction sadienne, qui doit sans cesse nous interroger sur les principes de la réalité.

Sade se considérait comme un homme de théâtre et sa passion pour le genre dramatique était peut-être avant tout celle de la comédie : "Je suis enfoncé dans mon cabinet au milieu de Molière, Destouches, Marivaux, Boissy, Regnard, que je regarde, considère, admire, et que je n'atteins jamais ${ }^{75} »$. C'est sans doute moins la médiocrité de ces pièces qu'il faut retenir, qu'une volonté de déjouer les codes pour faire de la comédie le lieu d'expression de sa pensée singulière. Les femmes sont pour beaucoup dans ce détournement des règles et des ressorts du comique. Sade ne prétend pas en faire des caractères de théâtre : ces personnages comiques se présentent à nous comme des entités philosophiques, porteuses d'un discours subversif cher au marquis - sur le mariage, les lois sociales, la place des femmes. La féminité est ici étroitement liée à la construction du comique sadien : ces femmes exemptes de ridicule, bien loin des restrictions bienséantes et vraisemblables du genre, se chargent d'exprimer le vice et déplacent la comédie vers l'utopie, non sans une certaine ironie de la part du dramaturge.

71. Ibid., acte II, sc. vI, p. 340.

72. Ibid., acte II, sc. X, p. 352.

73. Ibid., acte II, sc. XI, p. 354.

74. Avant-propos des Jumelles, op. cit., p. 286.

75. Correspondance inédite du marquis de Sade, publiée par Paul Bourdin, Paris, Librairie de France, 1929, p. 289. 
Ces figures féminines fonctionnent mal, en tant que personnages comiques, tant elles sont peu représentables sur scène et s'écartent des exigences théâtrales et des principes moraux. Si elles trahissent les ambitions philosophiques du marquis et une nouvelle manière de rire au théâtre, le défi de ce passionné de théâtre se change en gageure. On comprend mieux pourquoi Sade s'exclamait : « Le maudit métier que celui de faire des comédies ${ }^{76}$ ! » Déjouer les codes de la féminité et de la dramaturgie comique devait le priver de succès et laisser dans l'ombre sa carrière d'auteur dramatique.

Bénédicte Prot

Université de Lorraine, Université de Fribourg

76. Ibid., p. 283. 